\title{
Herba Scutellaria Barbata
}

National Cancer Institute

\section{Source}

National Cancer Institute. Herba Scutellaria Barbata. NCI Thesaurus. Code C2661.

A Chinese herb isolated from the plant Scutellaria barbata D. Don (Lamiaceae) with potential antineoplastic activity. Containing the antioxidant flavone scutellarin, herba Scutellaria barbata has been shown to induce apoptosis of ovarian and breast tumor cells in vitro. 